\title{
Of stem cells and ethics
}

\section{Advances in stem cell research offer unprecedented insights into human biology and opportunities for clinical translation. They also raise many questions with social and ethical implications.}

Owing to their unique ability to maintain, replenish and repair all tissue types, stem cells hold unparalleled promise for treating diseases outside the reach of conventional medicine. Remarkable strides have been made to translate basic research to the clinic following the path of blood stem cell transplantations, which are now an accepted standard of care. Clinical trials on a wide spectrum of conditions, using a variety of stem cell populations are now underway. Among them are several FDAapproved trials to treat patients with age-related macular degeneration with retinal pigment epithelium (RPE) derived from allogeneic human embryonic stem cells (ESCs). Preliminary results suggest favourable outcomes and an acceptable safety profile, with some adverse effects relating to the use of immunosuppressants. Earlier this year a milestone study (N. Engl. J. Med. 376, 1038-1046; 2017) reported the successful integration of autologous induced pluripotent stem cell (iPSC)-derived RPE cells in a patient with macular degeneration. Autologous cells have a low risk of immune rejection, and concerns about potential tumorigenicity of iPSC-derived cells were not substantiated during the trial. Thorough characterization of patient-derived cell lines used for transplantation led to one patient's exclusion after detection of genetic changes in the iPSCs. Another study this year described the complete reconstruction of the epidermis of a patient with epidermolysis bullosa by transplanting autologous transgenic epidermal stem cells (Nature http://doi.org/cf62). Additional trials on diabetes, ALS, Alzheimer's, Parkinson's disease and other conditions are ongoing. Counterbalancing the renewed excitement about the potential of stem cell therapies is the requirement that translational efforts rely on rigorous preclinical research to ensure the safety and efficacy of treatments.

Strong ethical considerations also weigh on research not yet in the clinic with clear potential for disease modelling and organ transplantation. Organoids are three-dimensional cellular microstructures that partly mimic the organization and function of an organ and can be generated from resident stem cells, ESCs and iPSCs. They can be readily established from biopsy samples, banked and manipulated, and have thus been proposed as ex vivo platforms for personalized treatments and source cells for gene correction and tissue transplantation. The range of possible uses raises many questions for the tissue donor, among them ensuring the anonymity of the tissue, ownership, long-term storage and manipulation. With rising interest from biotech and pharmaceutical companies, strategies for biobanking, commercialization and global distribution also require ethical review. Organoids derived from human PSCs do not yet achieve the functional maturation of their in vivo counterparts, often resembling early developmental stages instead of adult tissues. The extent of maturation that should be permitted is another question that arises from research of this kind.

Similar to organoids, gastruloids are PSC-derived, self-organizing structures that mimic key features of human gastrulation. Were such structures to be developed to some functional parity with human embryos, possible amendments to their regulatory status would be required (Development 144, 942-945; 2017). As of yet, the legal perspective on gastruloids is under discussion. Beyond organoids, research on donated human embryos continues to raise vigorous debate. The '14-day rule', a regulatory limit stated in guidelines issued by the International Society for Stem Cell Research (ISSCR) and reflected in the laws of at least 12 countries, mandates that research on human embryos be limited to the first two weeks after fertilization, and terminated at the emergence of the primitive streak, a precursor of the brain and spinal cord (Nature 533, 169-171; 2016). Until recently, human embryos could not be maintained in culture for substantially longer than a week. However, last year, two reports demonstrating sustained in vitro culture of human embryos for up to 13 days (Nat. Cell Biol. 18, 700-708; 2016 and Nature 533, 251-254; 2016) re-ignited discussions about whether this rule should remain firm in light of advanced technology, with no changes decided as yet.

This year also saw remarkable advances in human germline editing that opened up pressing ethical questions (Nature 549, 307; 2017), ranging from the possibility of correcting disease-initiating genetic mutations or using the technique to study early human development. Regulation of such research needs to take into account ethical procurement of embryos from in vitro fertilization treatments or their generation in the lab, using donated eggs and sperm. These diverse and unique aspects of stem cell and developmental studies necessitate strong guidelines to oversee and regulate any research in the area. In 2016, the ISSCR updated their guidelines (http://go.nature.com/2ADlppq) on clinical translation of stem cell research to ensure appropriate rigor, transparency and integrity in all stages of research and clinical application. Researchers must ensure ethical provenance of human biomaterials used, which mandates informed consent from donors regarding the proposed use and re-use of materials. The ISSCR recommends that research involving human embryos be subject to review, approval and monitoring by a specialized human embryo research oversight process.

At Nature Cell Biology, authors are asked to declare ethical compliance and confirm that approval of an ethics committee and informed consent were obtained for all studies involving human research participants or samples (including PSC lines, embryos and gametes), as outlined in our policy (http://go.nature.com/2xiGR4g). We are committed to safeguarding the scientific and ethical integrity of the research we publish, and thus in addition to strict editorial oversight of policy compliance, all studies involving human embryos are also reviewed by bioethicists. Over the past decade, a collective effort has enhanced our knowledge about strengths and potential risks associated with stem cell treatments. This knowledge has started to bear fruit in the clinic and it is vital that we adhere to established regulations, and update them as needed to match scientific advances with an open, constructive dialogue. Researchers, regulatory bodies, funders, policy makers, patient advocates and publishers must work together to ensure that progress and clinical translation are not impeded, but that ethical considerations are taken into account. 